Return to the Manage Active Submissions page at http://spie.org/submissions/tasks.aspx and approve or disapprove this submission. Your manuscript will not be published without this approval. Please contact author_help@spie.org with any questions or concerns.

\title{
VIRUS early installation and commissioning
}

Sarah E. Tuttle ${ }^{\mathrm{a}}$, Gary J. Hill ${ }^{\mathrm{a}}$, Brian L. Vattiat ${ }^{\mathrm{a}}$, Hanshin Lee ${ }^{\mathrm{a}}$, Niv Drory ${ }^{\mathrm{a}}$, Andreas Kelz ${ }^{\mathrm{b}}$, Jason Ramsey ${ }^{\mathrm{a}}$, Trent Peterson ${ }^{\mathrm{a}}$, Eva Noyola ${ }^{\mathrm{a}}$, Darren L. DePoy ${ }^{\mathrm{c}}$, Jennifer L. Marshall ${ }^{\mathrm{d}}$, Taylor S. Chonis ${ }^{\mathrm{a}}$, Gavin Dalton ${ }^{\mathrm{d}}$, Maximilian Fabricius ${ }^{\mathrm{e}}$, Daniel Farrow ${ }^{\mathrm{e}}$, John M. Good ${ }^{\mathrm{a}}$,

Dionne M. Haynes ${ }^{\mathrm{b}}$, Briana Indahl ${ }^{\mathrm{a}}$, Thomas Jahn ${ }^{\mathrm{b}}$, Hermanus Kriel ${ }^{\mathrm{a}}$, Harald Nicklas ${ }^{\mathrm{f}}$,

Francesco Montesano ${ }^{\mathrm{e}}$, Travis Prochaska ${ }^{\mathrm{c}}$, Richard D. Allen ${ }^{\mathrm{c}}$, Martin Landriau ${ }^{\mathrm{a}}$, Philip J. MacQueen $^{\mathrm{a}}$, Martin M. Roth ${ }^{\mathrm{b}}$, Richard Savage ${ }^{\mathrm{a}}$, and Jan M. Snigula ${ }^{\mathrm{e}}$

a McDonald Observatory, University of Texas at Austin, 2515 Speedway, Stop C1402, Austin, Texas, 78712, United States

beibniz-Institut für Astrophysik Potsdam (AIP), An der Sternwarte 16, 14482 Potsdam, Germany

'Texas A\&M University, Department of Physics and Astronomy, 4242 TAMU, College Station, TX 77843-4242, United States

${ }^{\mathrm{d}}$ Department of Physics, Oxford University, United Kingdom

eMax-Planck Institut für Extraterrestrische Physik (MPE), Germany

${ }^{\mathrm{f}}$ Georg-August-Univ., Göttingen, Germany

\begin{abstract}
VIRUS is a massively replicated spectrograph built for HETDEX, the Hobby Eberly Telescope Dark Energy Experiment. It consists of 156 channels within 78 units fed by 34944 fibers over the 22 arcminute field of the upgraded HET. VIRUS covers a relatively narrow bandpass $(350-550 \mathrm{~nm})$ at low resolution $(R \sim 700)$ to target the emission of Lyman-alpha emitters (LAEs) for HETDEX. VIRUS is a first demonstration of industrial style assembly line replication in optical astronomy.

Installation and testing of VIRUS units began in November of 2015. This winter we celebrated the first on sky instrument activity of the upgraded HET, using a VIRUS unit and LRS2-R (the upgraded facility Low Resolution Spectrograph for the HET). Here we describe progress in VIRUS installation and commissioning through June 2016. We include early sky data obtained to characterize spectrograph performance and on sky performance of the newly upgraded HET. As part of the instrumentation for first science light at the HET, the IFU fed spectrographs were used to test a full range of telescope system functionality including the field calibration unit (FCU). We also use placement of strategic IFUs to map the new HET field to the fiber placement, and demonstrate actuation of the dithering mechanism key to HETDEX observations.
\end{abstract}

Keywords: Spectrographs: Integral Field, Spectrographs: VIRUS, Spectrographs: performance, Telescopes: Hobby-Eberly

\section{INTRODUCTION}

VIRUS is the massively replicated spectrograph being built and installed at the newly upgraded Hobby Eberly Telescope (HET) for HETDEX - The Hobby Eberly Telescope Dark Energy eXperiment(1-3). VIRUS consists of 156 identical spectrograph channels contained in 78 identical units $(4,5)$. Each unit is fed by 448 fibers. In total, each VIRUS exposure contains 34944 spectra. Many of these spectra will be primarily sky, but we also expect to observe over a million Lyman alpha emitters (LAEs) to constrain dark energy in the era of star formation $(1.9 \leq z \leq 3.5)$. The blind survey will be conducted over two fields and at three years as a baseline. It is expected to begin once the full array is installed. The blind survey will also contain stars and galaxies that will provide an exciting catalog for further scientific discoveries.

Further author information: (Send correspondence to S. Tuttle.)

E-mail: tuttle@utexas.edu 
Return to the Manage Active Submissions page at http://spie.org/submissions/tasks.aspx and approve or disapprove this submission. Your manuscript will not be published without this approval. Please contact author_help@spie.org with any questions or concerns.

\section{SPECTROGRAPH DESCRIPTION}

The VIRUS spectrographs can be found discussed in detail in several past proceedings (6-8). Here we present an abbreviated version for context.

The spectrographs are based on a simple Schmidt camera. The camera and collimator are split mechanically for assembly and ease of repair. A VPH grating is used for diffraction(9). Two identical optical channels are contained in a single VIRUS unit to reduce the overall complexity of the VIRUS system.

The VIRUS spectrographs were designed to be simple - a limited bandpass (200nm) (based on science requirements), with no moving parts, VPH gratings, and off the shelf CCD detectors. The design uses as many reflective elements as possible, except for the grating and two aspheric correctors (a field flattener in front of the $\mathrm{CCD}$ and a corrector plate that doubles as the vacuum window for the camera cryostat).

\section{OVERVIEW OF SPECTROGRAPH ASSEMBLY AND ALIGNMENT}

Assembly of VIRUS units was conducted at the University of Texas, Austin (cameras \& collimators) and Texas A\&M University (TAMU)(collimators). This process has been ongoing in "assembly line" mode for just over two years. Although aiming for a truly identical process, there have been small changes as various issues arose. The first several spectrographs were at some level used to debug the process. We have also made small changes at our current pause in assembly to assess contamination issues with some detectors.

The design of the VIRUS units was driven by several principles including relative ease of assembly, a low level of precision needed for first assembly steps, and repeatability of the process. This allowed students to be involved at several stages of the work. It also meant that assembly shouldn't strongly depend on a single person. This was overall found to be true, although the copper cryogenic links required epoxying which was less repeatable than originally expected.

Alignment was done at UT using an in-situ system that allowed adjustment of the camera mirror under vacuum with a cooled detector $(10,11)$. The system was taken through focus and then adjusted to produce the best alignment. Initial plans were to independently align the camera and collimator to allow a "swappable" focus, to aid in possible maintenence later in the instrument's life. However, we found that removing that requirement and "permanently" mating cameras with collimators gave a marked improvement in the alignment of each unit.

\section{CHARACTERIZATION}

It is crucial that we characterize each spectrograph before deploying it to the telescope. This step is both a check and a record. We want to ensure that each unit is meeting specifications and provide a baseline for any possible change while the spectrographs are in service. The system is designed not to change, but issues with some of the detectors has motivated small changes to enable in situ monitoring of units installed at the telescope.

Each spectrograph unit has pixel flats (exposed with a relatively flat white spectrum from an LDLS (Laser Driven Light Source) and fiber flats (using Mercury Cadmium) taken, as well as darks. We measure an array of system parameters including gain and read noise, as well as checking for any structure on the detector, charge transfer efficiency issues, and a wavelength solution. A lab calibration unit (LCU) was built to mimic the FCU (Field Calibration Unit) used at the observatory. Details of the characterization work can be found in proceedings from this meeting (12).

\section{INSTALLATION}

The first "VIRUS" installation was actually half of LRS2 (Low Resolution Spectrograph 2) - a separate instrument, but designed within the mechanical infrastructure and interface of VIRUS (13) as well as a VIRUS unit. The installation mechanically is identical, and the only difference is that in the end the system is wired into a separate VMUX/computer interface from VIRUS. This installation run occured over during November 2015.

VIRUS units are stored backfilled with nitrogen when not in use. Before shipping, the units are pumped out to vacuum. They are strapped into our shipping vehicles on top of foam for the 8 hour drive to McDonald observatory(figure 1). Upon arrival, each unit is pumped out again to "top off" vacuum and guarantee they will 
Return to the Manage Active Submissions page at http://spie.org/submissions/tasks.aspx and approve or disapprove this submission. Your manuscript will not be published without this approval. Please contact author_help@spie.org with any questions or concerns.

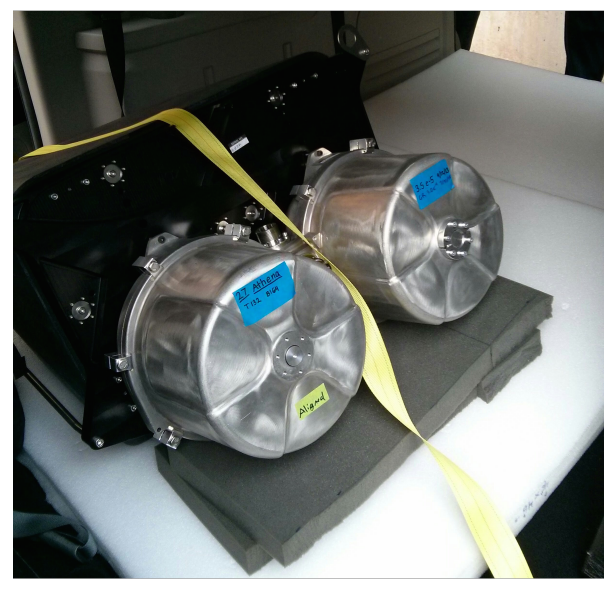

Figure 1. The first VIRUS unit packed for shipping in the back of a minivan. The drive to McDonald Observatory is just under 8 hours from UT Austin. (Image: S. Tuttle).

be ready to install the following morning. Three vacuum pumps are available to expeditate the process when several units are being installed as a block.

Our early installations were done with a smaller number of people, usually one or two in the air in the JLG/lift, bringing up the spectrograph using the dome crane (see figure 2). Then the same crew would climb to the back of the VIRUS enclosures to install the IFU fiber bundle. After a few installations it became obvious that having a second team of two on the enclsoures during the install considerably speeds up the process. Team 1 runs the JLG and crane, and Team 2 does all the enclosure work on the back.

Team 1 runs the crane to bring the spectrograph up to its position in the enclosure(14). The spectrograph slides along rails and is locked into position with a clamp (Team 2 confirms, as the clamp is in the back of the enclosures). Team 1 cables up the controller and confirms that communications are working. This is crucial as we need to be able to monitor the cooling of the detectors once the LN2 bayonet is installed. Once communications are up, the bayonet is brought down from its stowed position and locked into the spectrograph. The connection to the system is flexible conduit, which is crucial for the design. This does require care in positioning the connection for LN2 to flow steadily. Failure to position the tubing correctly is easily observed when the spectrograph system doesn't cool correctly. Usually a slight rearrangement of the bayonet system is all that is required.

Once the spectrograph is cooling, Team 2 seats the fibers using the kinematic mounts and bolts the slit plate into position. Both teams then close up the outer doors of the enclosure (front and back) to keep the system clean and light tight (figure 3).

\section{COMMISSIONING}

Commissioning of the ever-growing VIRUS array is ongoing. Units are integrated into observations as soon as they are cooled. This is one of the benefits of the multiplexed system - "VIRUS" as an instrument is in service almost regardless of the number of units available. The full array is required for the dark energy science the system is designed for. But work to debug the hardware infrastructure and software pipeline, as well as other science targets, doesn't necessarily require the full complement of spectrographs. IFUs are being installed contiguously to provide an ever growing field (as can be seen in figure 4). This allows for the partially completed instrument to be used for science applications that do not neccessarily require the large field. 
Please verify that (1) all pages are present, (2) all figures are correct, (3) all fonts and special characters are correct, and (4) all text and figures fit within the red margin lines shown on this review document. Complete formatting information is available at http://SPIE.org/manuscripts

Return to the Manage Active Submissions page at http://spie.org/submissions/tasks.aspx and approve or disapprove this submission. Your manuscript will not be published without this approval. Please contact author_help@spie.org with any questions or concerns.
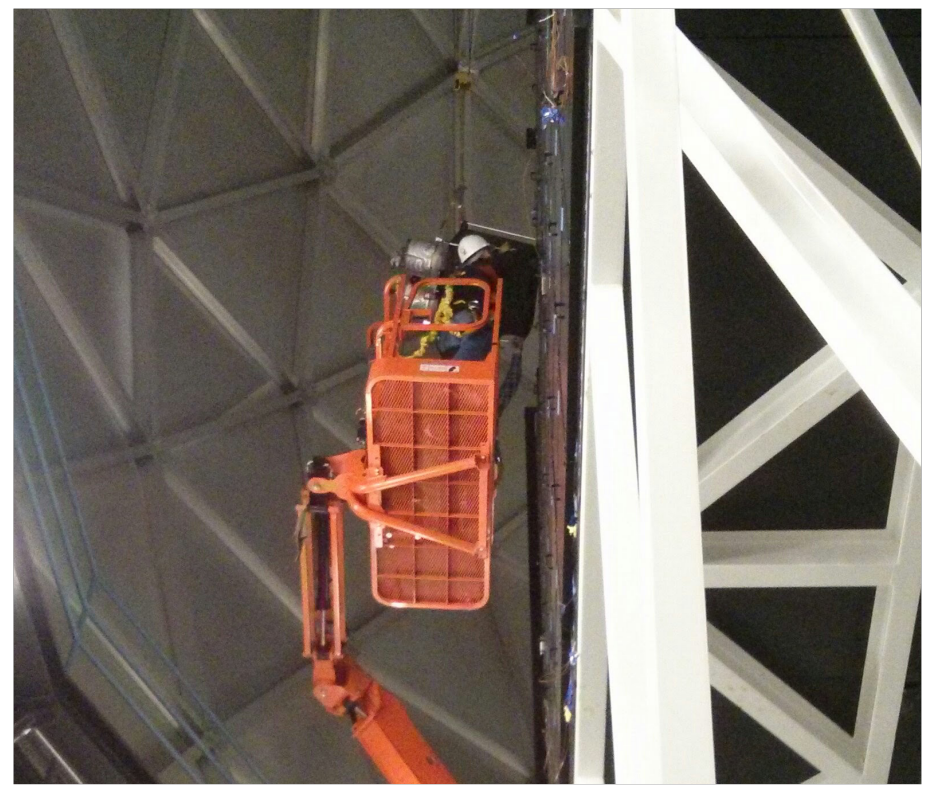

Figure 2. Sliding a VIRUS unit into position from the JLG/lift. The weight of the unit is born by the dome crane seen overhead.

9908 - 55 V. 2 (p.4 of 7) / Color: No / Format: Letter / Date: 7/20/2016 10:26:27 PM 
Return to the Manage Active Submissions page at http://spie.org/submissions/tasks.aspx and approve or disapprove this submission. Your manuscript will not be published without this approval. Please contact author help@spie.org with any questions or concerns.

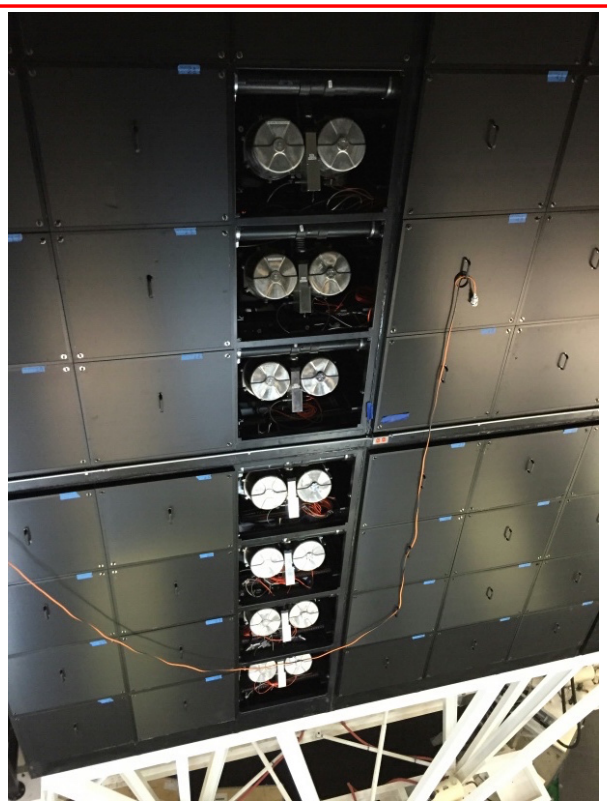

Figure 3. A full row of eight installed VIRUS units with the enclosure doors removed during installation. The VIRUS support structure (white weldment) is seen riding the air bearings at the bottom of the image. The black pipes seen above each unit remove heat from the controllers (seen between the cylinders of each unit.) (Image: T. Peterson)

Early work with commissioning has been to map the installed fiber bundle positions on sky and confirm astrometry against known SDSS fields. It has been important to confirm we could reconstruct the field accurately (and that we are mapping detector to sky correctly).
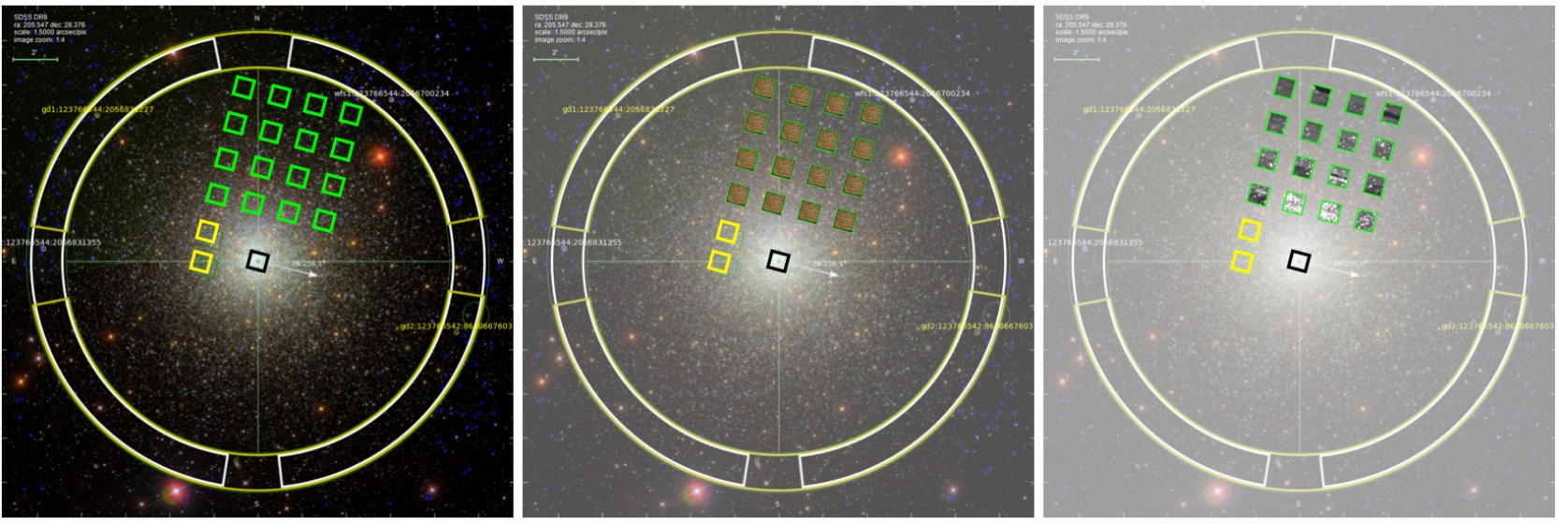

Figure 4. Left: SDSS target cluster with overlay of LRS2 (yellow) and VIRUS (green) IFU positions from VDAT (VIRUS Data Analysis Tool). Center: Fiber images populating VIRUS IFUs. Right: Reconstructed image from VIRUS data.

\section{IN SERVICE SPECTROGRAPH MONITORING}

A pixel flat system is being built for deployment at the observatory to allow for occasional pixel flats to be taken without removing the spectrographs (the fiber heads would have to be taken out briefly for the test.) This is particularly useful while questions remain about long term detector stability (as a result of the contamination issue discussed earlier). The whole system/spectrograph throughput will also be monitored through VDAT, for 
Return to the Manage Active Submissions page at http://spie.org/submissions/tasks.aspx and approve or disapprove this submission. Your manuscript will not be published without this approval. Please contact author_help@spie.org with any questions or concerns.

possible broken/damaged fibers as well as other issues (such as primary reflectivity changes). The FCU (facility calibration unit) is used at least once a night to provide fiber flats and measure any relative changes.

\section{NEXT STEPS}

VIRUS will continue to grow over the next year. As units are built and rehabilitated they will be characterized and shipped out to the mountain to join the array. Environmental stability in the enclosures is currently being tuned, including temperature/airflow as well as electrical/grounding paths. Current work on the software and hardware infrastructure means by the time the array is fully populated with 78 units (156 channels) the system should be fully debugged and ready for HETDEX survey and other work.

\section{ACKNOWLEDGMENTS}

HETDEX is run by the University of Texas at Austin McDonald Observatory and Department of Astronomy with participation from the Ludwig-Maximilians- Universitt Mnchen, (LMU) Max-Planck- Institut fr Extraterrestriche-Physik (MPE), Leibniz-Institut fr Astrophysik Potsdam (AIP), Texas Aamp;M University (TAMU), Pennsylvania State University (PSU), Institut fr Astrophysik Gttingen (IAG), University of Oxford, Max-Planck- Institut fr Astrophysik (MPA) and The University of Tokyo. In addition to Institutional support, HETDEX is funded by the National Science Foundation (grant AST-0926815), the State of Texas, the US Air Force (AFRL FA9451-04- 2-0355), by the Texas Norman Hackerman Advanced Research Program under grants 003658-0005- 2006 and 003658-0295- 2007, and by generous support from private individuals and foundations.

Financial support for innoFSPEC Potsdam of the German BMBF program Unternehmen Region (grant no. 03Z2AN11), and of Land Brandenburg, MWFK, is gratefully acknowledged. We also acknowledge support by the German BMI program Wirtschaft trifft Wissenschaft, grant no. 03WWBB105.

We thank the staffs of McDonald Observatory, HET, AIP, MPE, TAMU, IAG, Oxford University Department of Physics, the University of Texas Center for Electromechanics, and the University of Arizona College of Optical Sciences for their contributions to the development of the HET WFU and VIRUS.

\section{REFERENCES}

[1] Booth, J. A., MacQueen, P. J., Good, J. M., Wesley, G. L., Hill, G. J., Palunas, P., Segura, P. R., and Calder, R. E., "The wide field upgrade for the Hobby-Eberly Telescope," in [Society of Photo-Optical Instrumentation Engineers (SPIE) Conference Series], 6267, 62673W (June 2006).

[2] Hill, G. J., Booth, J. A., Cornell, M. E., Good, J. M., Gebhardt, K., Kriel, H. J., Lee, H., Leck, R., Moreira, W., MacQueen, P. J., Perry, D. M., Rafal, M. D., Rafferty, T. H., Ramiller, C., Savage, R. D., Taylor, C. A., Vattiat, B. L., Ramsey, L. W., Beno, J. H., Beets, T. A., Esguerra, J. D., Häuser, M., Hayes, R. J., Heisler, J. T., Soukup, I. M., Zierer, J. J., Worthington, M. S., Mollison, N. T., Wardell, D. R., and Wedeking, G. A., "Current status of the Hobby-Eberly Telescope wide field upgrade," in [Ground-based and Airborne Telescopes IV], 8444, 84440K (Sept. 2012).

[3] Hill, G. J., Drory, N., Good, J., Lee, H., Vattiat, B., Kriel, H., Bryant, R., Elliot, L., Landriau, M., Leck, R., Perry, D., Ramsey, J., Savage, R., Allen, R. D., Damm, G., DePoy, D. L., Fowler, J., Gebhardt, K., Haeuser, M., MacQueen, P., Marshall, J. L., Martin, J., Prochaska, T., Ramsey, L. W., Rheault, J.-P., Shetrone, M., Schroeder Mrozinski, E., Tuttle, S. E., Cornell, M. E., Booth, J., and Moreira, W., "Deployment of the Hobby-Eberly Telescope wide field upgrade," in [Ground-based and Airborne Telescopes V], 9145, 914506 (July 2014).

[4] Hill, G. J., MacQueen, P. J., Palunas, P., Kelz, A., Roth, M. M., Gebhardt, K., and Grupp, F., "VIRUS: A hugely replicated integral field spectrograph for HETDEX," 50, 378-381 (June 2006).

[5] Hill, G. J., Tuttle, S. E., Lee, H., Vattiat, B. L., Cornell, M. E., DePoy, D. L., Drory, N., Fabricius, M. H., Kelz, A., Marshall, J. L., Murphy, J. D., Prochaska, T., Allen, R. D., Bender, R., Blanc, G., Chonis, T., Dalton, G., Gebhardt, K., Good, J., Haynes, D., Jahn, T., MacQueen, P. J., Rafal, M. D., Roth, M. M., Savage, R. D., and Snigula, J., "VIRUS: production of a massively replicated 33k fiber integral field spectrograph for the upgraded Hobby-Eberly Telescope," in [Ground-based and Airborne Instrumentation for Astronomy IV], 8446, 84460N (Sept. 2012). 
Return to the Manage Active Submissions page at http://spie.org/submissions/tasks.aspx and approve or disapprove this submission. Your manuscript will not be published without this approval. Please contact author_help@spie.org with any questions or concerns.

[6] Tuttle, S. E., Allen, R. D., Chonis, T. S., Cornell, M. E., DePoy, D. L., Hill, G. J., Lee, H., Marshall, J. L., Prochaska, T., Rafal, M. D., Savage, R. D., and Vattiat, B. L., "Initial results from VIRUS production spectrographs," in [Ground-based and Airborne Instrumentation for Astronomy IV], 8446, 84465S (Sept. 2012).

[7] Hill, G. J., Tuttle, S. E., Drory, N., Lee, H., Vattiat, B. L., DePoy, D. L., Marshall, J. L., Kelz, A., Haynes, D., Fabricius, M. H., Gebhardt, K., Allen, R. D., Anwad, H., Bender, R., Blanc, G., Chonis, T., Cornell, M. E., Dalton, G., Good, J., Jahn, T., Kriel, H., Landriau, M., MacQueen, P. J., Murphy, J. D., Peterson, T. W., Prochaska, T., Nicklas, H., Ramsey, J., Roth, M. M., Savage, R. D., and Snigula, J., "VIRUS: production and deployment of a massively replicated fiber integral field spectrograph for the upgraded Hobby-Eberly Telescope," in [Ground-based and Airborne Instrumentation for Astronomy V], 9147, 91470Q (July 2014).

[8] Tuttle, S. E., Hill, G. J., Lee, H., Vattiat, B., Noyola, E., Drory, N., Cornell, M., Peterson, T., Chonis, T., Allen, R., Dalton, G., DePoy, D., Edmonston, D., Fabricius, M., Haynes, D., Kelz, A., Landriau, M., Lesser, M., Leach, B., Marshall, J., Murphy, J., Perry, D., Prochaska, T., Ramsey, J., and Savage, R., "The construction, alignment, and installation of the VIRUS spectrograph," in [Ground-based and Airborne Instrumentation for Astronomy V], 9147, 91470R (July 2014).

[9] Chonis, T. S., Frantz, A., Hill, G. J., Clemens, J. C., Lee, H., Tuttle, S. E., Adams, J. J., Marshall, J. L., DePoy, D. L., and Prochaska, T., "Mass production of volume phase holographic gratings for the VIRUS spectrograph array," in [Advances in Optical and Mechanical Technologies for Telescopes and Instrumentation], 9151, 91511J (July 2014).

[10] Lee, H., Hill, G. J., Tuttle, S. E., and Vattiat, B. L., "Fine optical alignment correction of astronomical spectrographs via in-situ full-field moment-based wavefront sensing," in [Modern Technologies in Space- and Ground-based Telescopes and Instrumentation II], 8450, 84500V (Sept. 2012).

[11] Lee, H., Hill, G. J., Tuttle, S. E., Noyola, E., Peterson, T., and Vattiat, B. L., "Field application of momentbased wavefront sensing to in-situ alignment and image quality assessment of astronomical spectrographs: results and analysis of aligning VIRUS unit spectrographs," in [Advances in Optical and Mechanical Technologies for Telescopes and Instrumentation], 9151, $91513 \mathrm{O}$ (July 2014).

[12] Indahl, B., Hill, G. J., Drory, N., Gebhardt, K., Tuttle, S., Ramsey, J., Ziemann, G., Chonis, T., Peterson, T., Peterson, A., Vattiat, B., Li, H., and Hao, L., "'VIRUS Characterization Development and Results from First Batches of Delivered Units," in [Modern Technologies in Space- and Ground-based Telescopes and Instrumentation II], (2016).

[13] Chonis, T. S., Hill, G. J., Lee, H., Tuttle, S. E., and Vattiat, B. L., "LRS2: the new facility low resolution integral field spectrograph for the Hobby-Eberly telescope," in [Ground-based and Airborne Instrumentation for Astronomy V], 9147, 91470A (July 2014).

[14] Prochaska, T., Allen, R., Mondrik, N., Rheault, J. P., Sauseda, M., Boster, E., James, M., RodriguezPatino, M., Torres, G., Ham, J., Cook, E., Baker, D., DePoy, D. L., Marshall, J. L., Hill, G. J., Perry, D., Savage, R. D., Good, J. M., and Vattiat, B. L., "VIRUS instrument enclosures," in [Ground-based and Airborne Instrumentation for Astronomy V], 9147, 91476U (Aug. 2014). 